\title{
EVOLUCIÓN DE BOSQUES ESTACIONALES SECOS NEOTROPICA- LES, CHACO Y CERRADO EN EL NEÓGENO DEL CENTRO-NORTE DE ARGENTINA
}

\author{
LUISA MATILDE ANZÓTEGUI ${ }^{1}$ \\ SILVINA SUSANA GARRALLA ${ }^{1}$ \\ LILIA RENÉ MAUTINO ${ }^{1}$ \\ DARIÉN PRADO²
}

\begin{abstract}
${ }^{1}$ Centro de Ecología Aplicada del Litoral (CECOAL)-Consejo Nacional de Investigaciones Científicas y Técnicas (CONICET)-Universidad Nacional del Nordeste. Ruta 5, km 2,5, 3400 Corrientes, Argentina.

${ }^{2}$ Instituto de Investigaciones en Ciencias Agrarias de Rosario (IICAR)-Consejo Nacional de Investigaciones Científicas y Técnicas (CONICET), Facultad de Ciencias Agrarias, Universidad Nacional de Rosario. Ocampo y Esmeralda, 2000 Rosario, Santa Fe, Argentina.
\end{abstract}

Recibido: 13 de diciembre de 2018 - Aceptado: 12 de abril de 2019

Para citar este artículo: Luisa Matilde Anzótegui, Silvina Susana Garralla, Lilia René Mautino, Darién Prado (2019). Evolución de Bosques Estacionales Secos Neotropicales, Chaco y Cerrado en el Neógeno del centro-norte de Argentina. Publicación Electrónica de la Asociación Paleontológica Argentina 19 (1): 7-17.

Link a este artículo: http://dx.doi.org/10.5710/PEAPA.12.04.2019.279

DESPLAZARSE HACIA ABAJO PARA ACCEDER AL ARTÍCULO

Otros artículos en Publicación Electrónica de la APA 18(1):

\section{Aldo R. Prieto}

HISTORIA DE LA PALINOLOGÍA DEL CUATERNARIO EN LA ARGENTINA: UNA RESEÑA A 90 AÑOS DE SUS COMIENZOS

\section{Virginia Mancini et al.}

ANÁLISIS Y MÉTODOS PALEOECOLÓGICOS PARA LA RECONSTRUCCIÓN DE COMUNIDADES DE BOSQUE Y ESTEPAS DE PATAGONIA, ARGENTINA

Asociación Paleontológica Argentina Maipú $6451^{\circ}$ piso, C1006ACG, Buenos Aires República Argentina Tel/Fax (54-11) 4326-7563 Web: www.apaleontologica.org.ar

\section{Dominique Mourelle et al.}

ANÁLISIS PALINOLÓGICO ACTUAL Y DEL CUATERNARIO TARDÍO EN LA REGIÓN DE LOS CAMPOS (URUGUAY Y SUR DE BRASIL): ESTADO DE LAS INVESTIGACIONES, DIFICULTADES Y POTENCIALIDADES 


\title{
EVOLUCIÓN DE BOSQUES ESTACIONALES SECOS NEOTROPICALES, CHACO Y CERRADO EN EL NEÓGENO DEL CENTRO-NORTE DE ARGENTINA
}

\author{
LUISA MATILDE ANZÓTEGUI', SILVINA SUSANA GARRALLA, LILIA RENÉ MAUTINO Y DARIÉN PRADO²
}

${ }^{1}$ Centro de Ecología Aplicada del Litoral (CECOAL)-Consejo Nacional de Investigaciones Científicas y Técnicas (CONICET)-Universidad Nacional del Nordeste. Ruta 5, km 2,5,3400 Corrientes, Argentina.luisaanzotegui@yahoo.com.ar; silgabe2@yahoo.com.ar; liliamautino@yahoo.com.ar

${ }^{2}$ Instituto de Investigaciones en Ciencias Agrarias de Rosario (IICAR)-Consejo Nacional de Investigaciones Científicas y Técnicas (CONICET), Facultad de Ciencias Agrarias, Universidad Nacional de Rosario. Ocampo y Esmeralda, 2000 Rosario, Santa Fe, Argentina.dprado@unr.edu.ar

Resumen. El objetivo de esta contribución es la de analizar la flora leñosa fósil (arbórea y arbustiva) neógena del centro-norte de Argentina y relacionarla con la flora de los actuales Bosques Estacionales Secos Neotropicales (BSEN), del Chaco y del Cerrado, a fin de establecer su permanencia y extensión a través del tiempo. A partir de las especies fósiles (polen, impresiones de hojas y de frutos, leños y cutículas) provenientes de las Formaciones Paraná, Ituzaingó, Anta, San José, Chiquimil, Palo Pintado, Andalhuala, del Buey, Toro Negro, Sálicas, Arroyo Feliciano y el pozo YPF.SE.x-2 Los Horcones y de la Aloformación Playa del Zorro comprendidas en el lapso Mioceno Medio-Pleistoceno, se evidencia la existencia de taxones fósiles leñosos cuyos afines actuales más cercanos habrían sido antecesores de los BSEN, del Chaco y del Cerrado. De este análisis se seleccionaron 14 especies como integrantes de los BSEN y 12 del Chaco y del Cerrado. Esta asociación fósil mantuvo una distribución geográfica más austral de lo que manifiestan en el presente la vegetación de los BSEN y la del Cerrado, siendo la de los BSEN igualmente fragmentaria y disyunta como la actual, mientras que en la del Cerrado se produjo una retracción más pronunciada quedando confinados actualmente al planalto brasileño.

Palabras clave. Neógeno. Bosques estacionales secos. Chaco. Cerrado. Argentina.

Abstract. EVOLUTION OF SEASONALLY DRY NEOTROPICAL FORESTS, CHACO AND CERRADO IN THE NEOGENE OF THE NORTHERN-CENTER OF ARGENTINA. The aims of this paper are to analyze the woody fossil (arboreal and shrubby) flora of the center-north of Argentina and to relate it to the flora of the current Seasonally Dry Neotropical Forests (SDNF), Chaco and Cerrado, in order to establish its permanence and extension over time. The fossil species (pollen, impressions of leaves and fruits, woods and cuticles) coming from of Paraná, Ituzaingó, Anta, San José, Chiquimil, Palo Pintado, Andalhuala, del Buey, Toro Negro, Sálicas, Arroyo Feliciano formations, YPF.SE.X-2 Los Horcones oil well, and the Playa del Zorro Aloformation. They are included in the Middle Miocene to Pleistocene period. There is evidence of woody fossil taxa with predecessors of the SDNF, Chaco and Cerrado. From this analysis, 14 species were selected as members of SDNF, 12 of Chaco and Cerrado. This fossil association maintained a more austral geographic distribution than the present vegetation of the SDNF and Cerrado. The fossil vegetation related to SDNF was equally fragmentary and disjunct, while the Cerrado had a more pronounced retraction, being confined to the Brazilian planalto.

Key words. Neogene. Seasonally dry neotropical forests. Chaco. Cerrado. Argentina.

Los trabajos paleoflorísticos del Neógeno del norte de Argentina mencionados en Garralla et al. (2016) dan a conocer una vasta lista de especies fósiles halladas en diversas localidades y formaciones (Fig. 1.1). A partir de ellos, se elaboró una base de datos integrada por 438 especies fósiles del Mioceno-Pleistoceno provenientes de las formaciones Paraná, Ituzaingó, Anta, San José, Chiquimil, Palo Pintado, Andalhuala, del Buey, Pozo YPF.SE.x-2 Los Horcones y de la Aloformación Playa del Zorro. Ante las reiteradas mencio- nes en Brea y Franco (2013), Brea et al. (2013); Franco y Brea (2008, 2009, 2010, 2013); Franco (2009, 2017); y Franco et al. (2013) de taxones del Mioceno-Pleistoceno del noreste argentino como posibles integrantes de los actuales Bosques Secos Estacionales Neotropicales (BSEN), y considerando la paleoflora censada por las autoras (Anzótegui, Garralla y Mautino) para el norte de Argentina, en el presente trabajo se encara la revisión de la paleoflora arbórea y arbustiva con el fin de hallar los posibles integrantes 
de este tipo de asociación vegetal. Por otra parte, Prado corroboró y señaló otros taxones que podrían haber formado parte de asociaciones vegetales semejantes a las actuales del Chaco (Prado, 1993a,b; Mogni et al., 2015 y DRYFLOR, 2017) y Cerrado (Ratter et al., 1997, 2003), estrechamente relacionadas a los BSEN. Por lo tanto, el objetivo de esta contribución es el de analizar la flora leñosa fósil (arbórea y arbustiva) neógena del centro-norte de Argentina y relacionarla con la flora característica de los actuales Bosques Estacionales Secos Neotropicales y otras unidades vegetales vecinas (Chaco y Cerrado), a fin de establecer su permanencia y extensión a través del tiempo.

El concepto actual de los BSEN (Bosques Secos Estacionales Neotropicales) abarca un tipo de vegetación leñosa, mayoritariamente caducifolia, con una fuerte estacionalidad climática, producto de una estación seca de duración variable en los trópicos de Sudamérica (DRYFLOR, 2016). Se trata de ecosistemas arbóreos y arbustivos, con el dosel relativamente continuo y caducifolio, con precipitaciones que oscilan desde 350 a $1.000 \mathrm{~mm} /$ año y una notable estación seca de 3 a 6 meses. Se establecen sobre suelos pedregosos y fértiles, con pH ligeramente ácido a alcalino (valor 6 ó mayor). En el continente sudamericano se manifiestan por una serie de fragmentos que atraviesan el continente en forma de arco o herradura, con la convexidad meridional que se extiende desde las Caatingas del NE del Brasil, hacia el sur-este en el sector de las antiguas misiones jesuíticas de Brasil, Paraguay y Argentina; hacia el sur-oeste en el Bosque Pedemontano Subandino de las Yungas de Argentina y Bolivia, ambos sectores conectados a través de la Chiquitanía boliviana y nor-paraguaya, para llegar finalmente a los valles secos interandinos de Bolivia y Perú (Prado y Gibbs, 1993; Pennington et al., 2000, 2004; Prado, 2000; Mogni et al., 2015).

En esta concepción de los BSEN, quedan claramente excluidos los bosques y sabanas del Chaco s.s. de Bolivia, Paraguay y Argentina, que abarcan formaciones subtropicales a templadas sujetas a fuertes heladas anuales, sobre suelos arcillosos salino-alcalinos y con una flora notoriamente distinta de los BSEN (Prado, 1993a, 2000; Prado y Gibbs, 1993; Pennington et al., 2000, 2004; Caetano et al., 2008; Mogni et al., 2015; DRYFLOR, 2016). También son muy diferentes las extensas sabanas graminosas del Ce- rrado del centro del Brasil, y pequeñas intrusiones en el NE de Bolivia y del Paraguay, con suelos ferralíticos distróficos y muy ácidos, con altos contenidos de aluminio (que se acumula en el follaje de sus plantas), por lo tanto una flora muy diferente de los dos grandes biomas precedentes y muy rica en endemismos (Ratter et al., 1997, 2003; Pennington et al., 2000; Furley, 2007).

\section{MATERIALES Y MÉTODOS}

Las especies fósiles utilizadas provienen de una base de datos integrada por 438 especies fósiles obtenidas a partir de estudios de polen, leños, cutículas, impresiones de hojas y frutos (Garralla et al., 2016). A dicha base, se sumaron los taxones provenientes de las formaciones Toro Negro (Martínez y Rodríguez-Brizuela, 2011), Sálicas (Pujana et al., 2014), Arroyo Feliciano (Moya y Brea, 2015a,b; Moya et al., 2015, 2017) y El Palmar (Ramos et al., 2015). La edad y procedencia geográfica de las formaciones portadoras de las especies fósiles utilizadas se especifican en la Tabla 1. De esta base, se seleccionaron 84 taxones cuyos afines actuales son árboles o arbustos. Entre ellos, uno de los autores (D. Prado) identificó 14 integrantes de los BSEN (Prado, 2000; Mogni et al., 2015; DRYFLOR, 2016, 2017) y 12 de las unidades vegetales del Chaco (Prado, 1993a,b; Mogni et al., 2015 y DRYFLOR, 2017) y del Cerrado (Ratter et al., 1997, 2003; Pennington et al., 2000; Furley, 2007). Los afines actuales de los taxones fósiles, tales como, Syagrus sp. (Anzótegui y Lutz, 1988), Maytenus sp. (Franco, 2017), Sebastiania sp. (Anzótegui y Lutz, 1988) y Sorocea sp. (Franco, 2010) no están determinadas a nivel de especie, o sus afines actuales son numerosos, como es el caso de las Mirtáceas (Anzótegui, 2000), razón por la cual no fueron consideradas como integrantes de los BSEN. En el texto, las listas de especies se citan por su denominación actual, seguida por la fósil entre paréntesis y agrupadas, cuando corresponde, por la familia a la que pertenecen. En los casos donde la denominación de la especie actual y fósil es la misma, e.g., Tipuana tipu (Benth.) Kuntze 1898, solo se emplea la de la especie actual.

Para la denominación de las especies actuales se sigue a Zuloaga et al. (2008) y a Tropicos.org. 


\begin{tabular}{|c|c|c|}
\hline Formaciones & Edad & Provincia \\
\hline Paraná & Mioceno Tardío-Plioceno & Santa Fe, Entre Ríos \\
\hline Ituzaingó & Plioceno-Pleistoceno & Corrientes, Entre Ríos \\
\hline Arroyo Feliciano & Pleistoceno Tardío & Entre Ríos \\
\hline El Palmar & Pleistoceno Tardío & Entre Ríos \\
\hline Anta & Mioceno Temprano medio & Salta \\
\hline San José & Mioceno Medio & Tucumán, Catamarca, Salta \\
\hline Chiquimil & Mioceno Tardío & Catamarca, Tucumán \\
\hline Aloformación Playa del Zorro & Mioceno Tardío & Catamarca \\
\hline del Buey & Mioceno Medio & La Rioja \\
\hline Sálicas & Mioceno Tardío & La Rioja \\
\hline Toro Negro, Grupo Vinchina & Mioceno Tardío-Plioceno & La Rioja \\
\hline Palo Pintado & Mioceno Tardío & Salta \\
\hline Andalhuala & Plioceno & Catamarca, Tucumán \\
\hline Pozo YPF.SE.x-2 Los Horcones & Mioceno Tardío? & Santiago del Estero \\
\hline
\end{tabular}

\section{RESULTADOS Y DISCUSIÓN}

Los taxones fósiles seleccionados en este trabajo integran la provincia Paleofitogeográfica Neotropical de Argentina (Barreda et al., 2007); forman (por comparación con los afines actuales) parte de los bosques de ribera sensu lato (selvas) y sabanas arboladas (con vegetación xerófila) del norte de Argentina, establecidos por Anzótegui y Horn (2011); Mautino y Anzótegui (2014); Garralla et al. (2016); Anzótegui et al. (2017). Ellos muestran los vínculos entre la vegetación del Neógeno y la de los actuales BSEN, la vegetación leñosa del Chaco y del Cerrado.

Las especies fósiles de bosques de ribera sensu lato (selva) son: Acacia visco Grisebach 1874 (Paraalbizioxylon caccavariae Martínez 2014), Erythrina dominguezii Hassler 1922, Pithecellobium diversifolium Bentham 1844, Anadenanthera colubrina (Vellozo) Brenan 1955 (Polliadopolenites sp., en Barreda y Caccavari, 1992; Anadenantheroxylon viIlaurquiense Brea, Aceñolaza y Zucol 2001), Tipuana tipu; Gleditsia amorphoides (Grisebach) Tauber 1892 (Gleditsioxylon riojana Martínez y Rodríguez-Brizuela 2011, Gleditsioxylon paramorfoides Franco y Brea 2013 y Margocolporites sp., en Mautino et al., 2017); Balfourodendron riedelianum (Engler) Engler 1896 (Rhoipites angurium (Partridge) Pocknall y Mildenhall 1984 y leño innominado en Franco et al., 2013); Jacaranda mimosifolia D. Don 1822 (Margocolporites cf. M. scabrosus Pocknall y Mildenhall 1984, en Mautino, 2010); Phyllostylon rhamnoides (Poisson) Tauber 1890 (Ulmipollenites undulosus Wolff 1934, en Frederiksen, 1988); Myracrodruon urundeuva Freire Allemão 1962; (Astroniunxylon portmanii Brea, Aceñolaza y Zucol 2001 y Astroniunxylon bonplandianum Franco 2009), Myracrodruon balansae (Engl.) Santin 1991 (Astroniumxylon parabalansae Franco y Brea 2008); Cedrela fissilis Vellozo 1835 (Cedrela fissiliformis Anzótegui y Horn 2011), Trichilia catigua Jussieu 1829; Ficus luschnathiana (Miquel) Miquel 1868 (Ficus tressensis Anzótegui 1998); Ruprechtia laxiflora Meissner 1855 (Ruprechtioxylon breae Franco 2017); Stryphonodendron macrostachyum Poepp 1845 (Stryphonodendron aff. macrostachyum y Políade tipo 1 , en Mautino, 2010), Machaerium aculeatum Raddi 1820 (Rhoipites exiguus Pocknall 1982); Qualea sp. (Qualeaxylon felicia- 
nensis Moya y Brea 2015).

De las sabanas arboladas: Acosmium subelegans (Mohlenbrock) Yakovlev 1969; Copernicia sp./Trithrinax sp., (en Anzótegui y Lutz, 1988); Schinopsis lorentzii (Grisebach) Engler 1881, Prosopis spp. (Prosopis sp. en Anzótegui y Herbst, 2004), Prosopis sp. 1 en Anzótegui et al., 2007b; Prosopisinoxylon anciborae Martínez 2010; Prosocolporites argentina Mautino y Anzótegui 2014; Prosopisinoxylon castroae Brea, Zucol y Patterer 2010; Prosopisinoxylon americanun Franco y Brea 2013; Cynophalla retusa (Grisebach) Cornejo e Iltis 2008 (Rhoipites karamuensis Pocknall 1985); Celtis sp. (Triorites orbiculatus McIntyre 1965) y Bulnesia retama (Gillies ex
Hooker et Arnott) Grisebach 1874.

Si se considera que desde el Mioceno Medio al Pleistoceno Tardío del norte de Argentina se citan 84 especies fósiles arbóreas y arbustivas, en tanto que solo 27 de ellas se mencionan en la vegetación actual de los BSEN, del Chaco y del Cerrado, se infiere que la vegetación fósil predominante del citado período no estaba conformada y delimitada fitogeográficamente como se encuentra actualmente. Por lo tanto dicha paleovegetación neógena puede ser considerada en su conjunto antecesora de las actuales como lo ya postulado por Anzótegui y Horn (2011) y Mautino y Anzótegui (2014).

TABLA 2 - Asociación leñosa del Mioceno-Pleistoceno del norte argentino cuyos afines actuales integran los BSEN. Formaciones que los contienen y edad

\begin{tabular}{|c|c|c|c|c|}
\hline \multirow{2}{*}{ Afin actual (sp.) } & \multirow{2}{*}{ Especies fósiles } & \multicolumn{2}{|c|}{ Formaciones } & \multirow{2}{*}{ Edad } \\
\hline & & $N E$ & NO & \\
\hline 1-Pithecelobium diversifolium & Pithecelobium diversifolium & & San José & MM \\
\hline 2-Balfourodendron riedelianum & $\begin{array}{l}\text { Rhoipites angurium, } \\
\text { Leño indet. }\end{array}$ & Ituzaingó & San José & $\begin{array}{l}M M \\
P-P\end{array}$ \\
\hline \multirow[t]{2}{*}{ 3-Anadenanthera colubrina } & Polliadopolenites $s p$. & & San José & MM \\
\hline & $\begin{array}{l}\text { Anadenantheroxylon } \\
\text { villaurquiense }\end{array}$ & $\begin{array}{l}\text { Paraná } \\
\text { Ituzaingó }\end{array}$ & Palo Pintado & $\begin{array}{l}M T \\
M T-P \\
P P\end{array}$ \\
\hline 4-Jacaranda mimosifolia & Margocolporites $c f$. M. scabrosus & & Chiquimil & MT \\
\hline 5-Phyllostilon rhamnoides & Ulmipollenites undulosus & & Chiquimil & MT \\
\hline 6-Tipuana tipu & Tipuana tipu & & $\begin{array}{l}\text { Chiquimil } \\
\text { Andalhuala }\end{array}$ & $\begin{array}{c}M T \\
P\end{array}$ \\
\hline 7-Myracrodruon urundeuva & $\begin{array}{l}\text { Astroniumxylon portmanii, } \\
\text { A. bonplandianum }\end{array}$ & $\begin{array}{l}\text { Paraná } \\
\text { Ituzaingó }\end{array}$ & & $\begin{array}{l}M T-P \\
P-P\end{array}$ \\
\hline 8-Cedrela fissilis & Cedrela fissiliformis & & Palo Pintado & MT \\
\hline 9-Ficus luschnathiana & Ficus tressensis & & San José & \\
\hline 10-Gleditsia amorphoides & $\begin{array}{l}\text { Gleditsioxylon riojana, } \\
\text { Margocolporites } s p ., \\
\text { G. paramorfoides }\end{array}$ & Ituzaingó & $\begin{array}{l}\text { Toro Negro } \\
\text { Palo Pintado }\end{array}$ & $\begin{array}{l}M T \\
M T \\
M T\end{array}$ \\
\hline 11-Myracrodruon balansae & Astroniumxylon parabalansae & $\begin{array}{l}\text { Ituzaingó } \\
\text { Paraná }\end{array}$ & & $\begin{array}{l}M T-P \\
P-P\end{array}$ \\
\hline 12-Trichilia catigua & Trichilia catigua & Ituzaingó & & $P-P$ \\
\hline 13-Ruprechtia laxiflora & Ruprechtioxylon breae & Ituzaingó & & $P-P$ \\
\hline 14-Parapiptadenia rigida & Menendoxylon piptadensis & El Palmar & & PT \\
\hline
\end{tabular}

NE, noreste; NO, noroeste; MM, Mioceno Medio; MT, Mioceno Tardí; MT-P, Mioceno Tardío-Plioceno; P, Plioceno; P-P, Plioceno-Pleistoceno. 
TABLA 3 - Asociación leñosa del Mioceno-Pleistoceno del norte argentino cuyos afines actuales integran las unidades vegetales del Cerrado y Chaco

\begin{tabular}{|c|c|c|c|c|c|}
\hline \multirow{2}{*}{ Afin actual (sp.) } & \multirow{2}{*}{ Especies fósiles } & \multicolumn{2}{|c|}{ Formaciones } & \multirow{2}{*}{ Edad } & \multirow{2}{*}{$\begin{array}{c}\text { Ecosistema } \\
\text { vegetal }\end{array}$} \\
\hline & & $N E$ & NO & & \\
\hline 1- Erythrina dominguezii & Erythrina dominguezii & & San José & $M M$ & Cerrado \\
\hline 2-Acosmium subelegans & Acosmium $c f$. subelegans & & & & \\
\hline 3-Qualea sp. & Qualeaxylon felicianensis & Arroyo Feliciano & & PT & \\
\hline \multirow[t]{2}{*}{ 4-Stryphonodendron macrostachyum } & Poliade Tipo 1 & & $\begin{array}{l}\text { San José } \\
\text { Chiquimil }\end{array}$ & $\begin{array}{l}M M \\
M T\end{array}$ & \\
\hline & $\begin{array}{l}\text { Stryphonodendron aff. } \\
\text { macrostachyum }\end{array}$ & $\begin{array}{l}\text { Paraná } \\
\text { Ituzaingó }\end{array}$ & & & \\
\hline 5-Machaerium aculeatum & Rhoipites exiguus & Paraná & $\begin{array}{l}\text { San José } \\
\text { Chiquimil }\end{array}$ & $\begin{array}{c}M M \\
M T \\
M T-P\end{array}$ & \\
\hline 6-Schinopsis lorentzii & Schinopsis lorentzii & & $\begin{array}{l}\text { San José } \\
\text { Alof. Playa }\end{array}$ & & Chaco \\
\hline 7-Copernicia $s p . /$ Trithrinax $s p$. & Copernicia $s p . /$ Trithrinax $s p$. & $\begin{array}{l}\text { Paraná } \\
\text { Ituzaingó }\end{array}$ & & $\begin{array}{l}M T-P \\
P-P\end{array}$ & \\
\hline 8-Capparis retusa & Rhoipites karamuensis & & $\begin{array}{l}\text { San José } \\
\text { Chiquimil }\end{array}$ & $\begin{array}{l}M M \\
M T\end{array}$ & \\
\hline 9-Celtis spp. & Celtis orbicularis & Paraná & $\begin{array}{l}\text { San José } \\
\text { Chiquimil }\end{array}$ & & \\
\hline \multirow[t]{4}{*}{ 10-Prosopis spp. } & $\begin{array}{l}\text { Prosocolporites argentina } \\
\text { Prosopis } s p \text {. }\end{array}$ & & San José & $M M$ & \\
\hline & Prosopis sp. 1 & & $\begin{array}{c}\text { San José } \\
\text { Alof. Playa } \\
\text { Chiquimil }\end{array}$ & $\begin{array}{l}M M \\
M T \\
M T\end{array}$ & \\
\hline & Prosopisinoxylon anciborae & & Sálicas & MT & \\
\hline & $\begin{array}{l}\text { Prosopisinoxylon americanun } \\
\text { Prosopisinoxylon castroae }\end{array}$ & $\begin{array}{l}\text { Ituzaingó } \\
\text { El Palmar } \\
\text { El Palmar }\end{array}$ & & $\begin{array}{c}P-P \\
P T \\
P T\end{array}$ & \\
\hline 11-Acacia visco & Paraalbizioxylon caccavariae & & Chiquimil & MT & \\
\hline 12-Bulnesia retama & Bulnesia $c f$ retama & & San José & $M M$ & \\
\hline
\end{tabular}

NE, noreste; NO, noroeste; MM, Mioceno Medio; MT, Mioceno Tardío; MT-P, Mioceno Tardío-Plioceno; P, Plioceno; PT, Pleistoceno Tardío; P-P, PliocenoPleistoceno.

\section{Antecesores fósiles de los BSEN}

La asociación leñosa cuyos afines actuales integran los BSEN está conformada por 14 especies fósiles que pertenecen a las siguientes familias: Fabaceae: Pithecellobium diversifolium, Anadenanthera colubrina, Tipuana tipu, Gleditsia amorphoides; Rutaceae: Balfourodendron riedelianum; Bignoniaceae: Jacaranda mimosifolia; Ulmaceae: Phyllostylon rhamnoides; Anacardiaceae: Myracrodruon urundeuva, Myracrodruon balansae; Meliaceae: Cedrela fissilis y Trichilia cati- gua; Moraceae: Ficus luschanathiana y Polygonaceae: Ruprechtia laxiflora (Tab. 2; Fig. 1).

Es de destacar que, en el Mioceno Medio (Fig. 1.2) Pithecelobium diversifolium, Ficus luschnathiana, Balfourodendron riedelianum y Anadenanthera colubrina ya estaban instaladas en la región del noroeste. Mientras que en el lapso Mioceno Tardío-Plioceno (Fig. 1.3) se registran tanto en el noroeste como en el noreste las siguientes especies: Jacaranda mimosifolia, Phyllostylon rhamnoides, Tipuana tipu, Myracrodruon 
urundeuva, Cedrela fissilis, Ficus Iuschnathiana, Gleditsia amorphoides y Anadenanthera colubrina, observándose en el noroeste una mayor representatividad. En el PliocenoPleistoceno (Fig. 1.4) solo se registran en el nordeste, Gleditsia amorphoides, Anadenanthera colubrina, Myracrodruon balansae, Trichilia catigua, Ruprechtia laxiflora y Parapiptadenia rigida.

Las especies Anadenanthera colubrina, Gleditsia amorphoides y Tipuana tipu son las más frecuentes en la región de estudio. Anadenanthera colubrina es la que posee mayor distribución temporal y areal, pues ya Barreda y Caccavari (1992) la hallaron en sedimentos de la Formación Chenque del Mioceno Temprano en la Patagonia argentina, en tanto que en el norte se la registra desde el Mioceno Medio al Plioceno-Pleistoceno en las formaciones San José, Palo Pintado, Paraná e Ituzaingó (Mautino, 2010; Franco y Brea, 2013; Horn, 2014; Fernández Pacella, 2015). Gleditsia amorphoides es citada en las formaciones Palo Pintado (Mautino et al., 2017), Toro Negro (Martínez y Rodríguez-Brizuela, 2011) e Ituzaingó (Franco y Brea, 2013) y Tipuana tipu en Chiquimil (Anzótegui et al., 2007a) y Andalhuala (Anzótegui et al., 2007b).

En el lapso estudiado, están representadas cinco especies de Fabaceae, dos de Anacardiaceae y Meliaceae y una de Bignoniaceae. La presencia de estas familias refleja la misma tendencia actual sugerida por Prado (1993a, 2000), Prado y Gibbs (1993), Pennington et al. (2000, 2004), Caetano et al. (2008), Mogni et al. (2015) y DRYFLOR (2016) para las asociaciones actuales de los BSEN.

Es de destacar que las especies Anadenanthera colubrina y Myracrodruon urundeuva, consideradas actualmente paradigmáticas de los BSEN por Prado y Gibbs (1993); Prado (2000); Pennington et al. (2000, 2004); Gosling et al. (2005); Mayle (2004); Mayle et al. (2004), Caetano et al. (2008) y Mogni et al. (2015), también integran la vegetación leñosa del Neógeno. Sin embargo, es Anadenanthera colubrina la que evidencia una mayor distribución y representación fósil en el norte argentino (en las provincias de Tucumán, Salta, Entre Ríos y Corrientes) en relación a Myracrodruon urundeuva, que solo presenta registro en la provincia de Entre Ríos (Tab. 2; Fig. 1). Actualmente, Anadenanthera colubrina con sus dos variedades colubrina y cebil cohabita en las regiones establecidas para los BSEN (Catamarca, Chaco, Córdoba, Co- rrientes, Entre Ríos, Formosa, Jujuy, Misiones, Salta, Santiago del Estero y Tucumán) mientras que Myracrodruon urundeuva se encuentra en las provincias de Chaco, Salta y Jujuy, extinguiéndose localmente para la provincia de Entre Ríos (Zuloaga et al., 2008).

Si bien Pithecelobium diversifolium crece en el nordeste de Brasil (Zuloaga et al., 2008), en el Mioceno Medio formaba parte de la vegetación del noroeste argentino (Formación San José), señalando una distribución más austral. Por otra parte, Ficus luschnathiana, Phyllostylon rhamnoides y Cedrela fissilis, actualmente confinadas al noreste de Argentina (Zuloaga et al., 2008), integraban en el Mioceno Medio y Tardío la vegetación fósil de los bosques de ribera del noroeste argentino. Estas especies, al igual que Myracrodruon urundeuva, se habrían extinguido localmente de las regiones donde fueron halladas como fósiles.

\section{Antecesores fósiles de las unidades vegetales de Chaco y Cerrado}

Las especies fósiles leñosas afines a las actuales unidades vegetales del Chaco y Cerrado y que a su vez guardan estrechas relaciones con los BSEN (Tab. 3; Fig. 2), son para el Chaco, Arecaceae: Copernicia sp./Trithrinax sp.; Anacardiaceae: Schinopsis lorentzii; Fabaceae: Prosopis spp. (Prosopis sp., Prosopis sp. 1, Prosopisinoxylon anciborae, Prosocolporites argentina, Prosopisinoxylon castroae y Prosopisinoxylon americanun); Acacia visco (Paraalbizioxylon caccavariae); Capparidaceae: Cynophalla retusa (Rhoipites karamuensis); Celtidaceae: Celtis sp. (Triorites orbiculatus) y Zygophyllaceae: Bulnesia retama. En tanto que, para el Cerrado son: Fabaceae: Erythrina dominguezii, Acosmium subelegans (Acosmium cf. subelegans), Stryphonodendron macrostachyum (Stryphonodendron aff. macrostachyum y Políade tipo 1), Machaerium aculeatum (Rhoipites exiguus) y Vochysiaceae: Qualea sp. (Qualeaxylon felicianensis).

La mayoría de las especies leñosas citadas datan del Mioceno Medio y se encuentran bien representadas hasta el Mioceno Tardío-Plioceno, decrecen hacia el Pleistoceno Tardío, quedando finalmente reducidas a las siguientes: Qualea sp. (Qualeaxylon felicianensis), Prosopis sp. (Prosopisinoxylon americanun, Prosopisinoxylon castroae), Copernicia sp./ Trithrinax sp. y Stryphonodendron macrostachyum (Stryphonodendron aff. macrostachyum), todas en el noreste. 
Por otra parte, las especies con mayor representación y amplia distribución areal son: Prosopis sp. (en 7 formaciones aflorantes en las provincias de Tucumán, Catamarca, La Rioja, Corrientes y Entre Ríos), Stryphonodendron macrostachyum (en 4 formaciones de las provincias de Tucumán, Catamarca, Corrientes y Entre Ríos), Machaerium aculeatum y Celtis sp. (en 3 formaciones de las provincias de Tucumán, Catamarca, Corrientes y Entre Ríos), Cynophalla retusa y Schinopsis lorentzii (en 2 formaciones de las provincias de Tucumán y Catamarca) (Tab. 3). De aquí se desprende, que las especies fósiles afines a las actuales integrantes de la vegetación leñosa del Chaco y del Cerrado tuvieron un re-
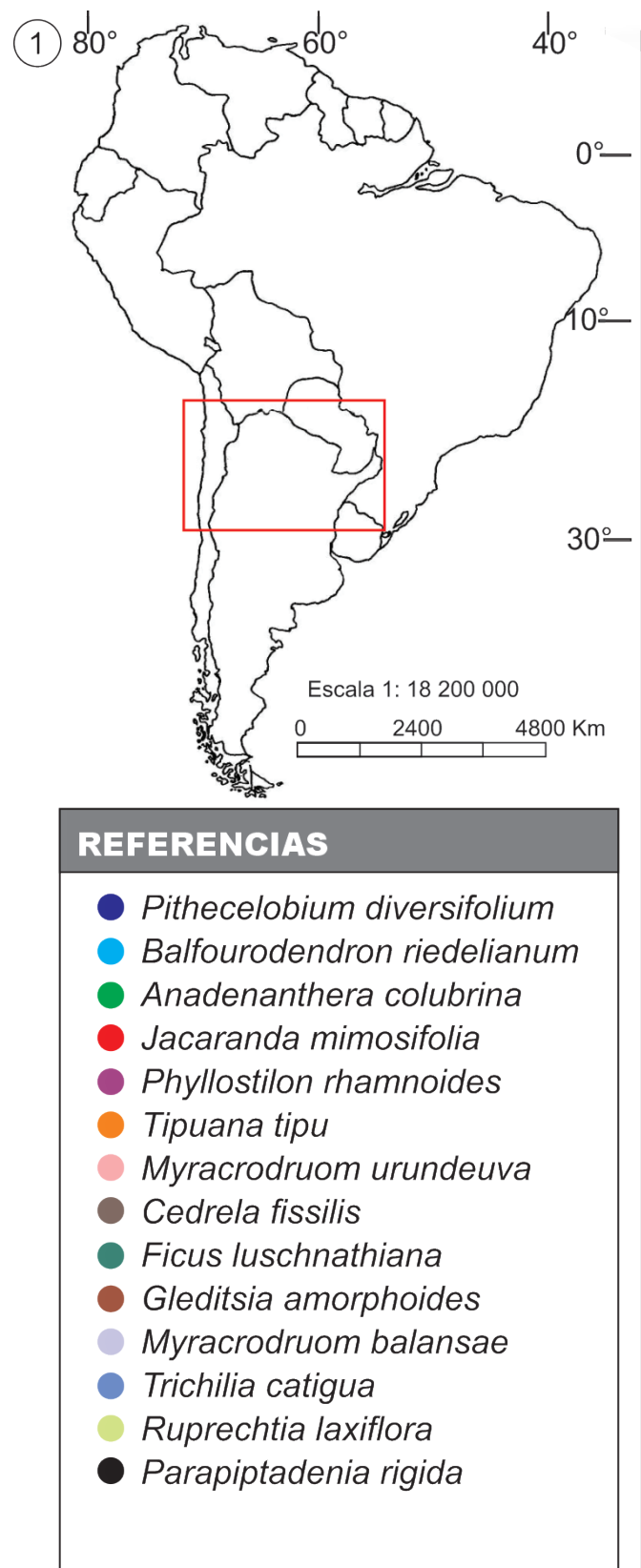
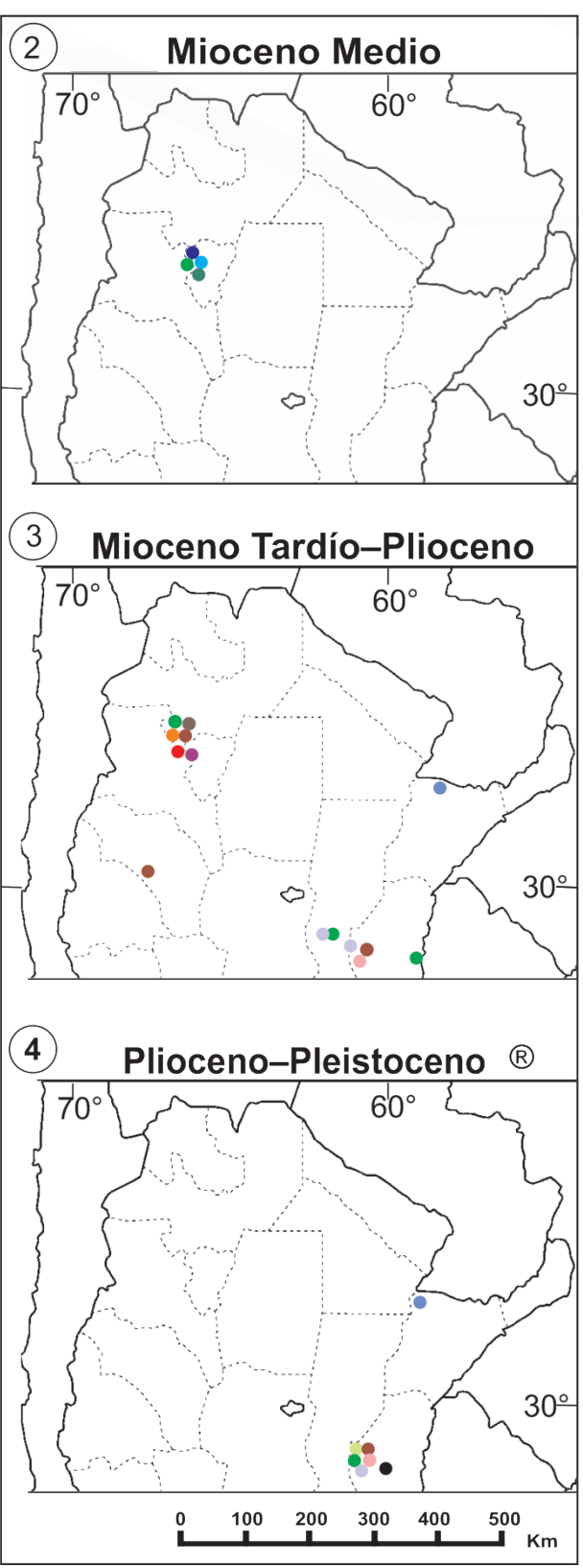

Figura 1. 1, Área generalizada de los yacimientos plantíferos neógenos de los BSEN (Bosques estacionales secos neotropicales). 2, Distribución de las especies fósiles en el Mioceno Medio. 3, Distribución de las especies fósiles en el Mioceno Tardío-Plioceno. 4, Distribución de las especies fósiles en el Pleistoceno. 


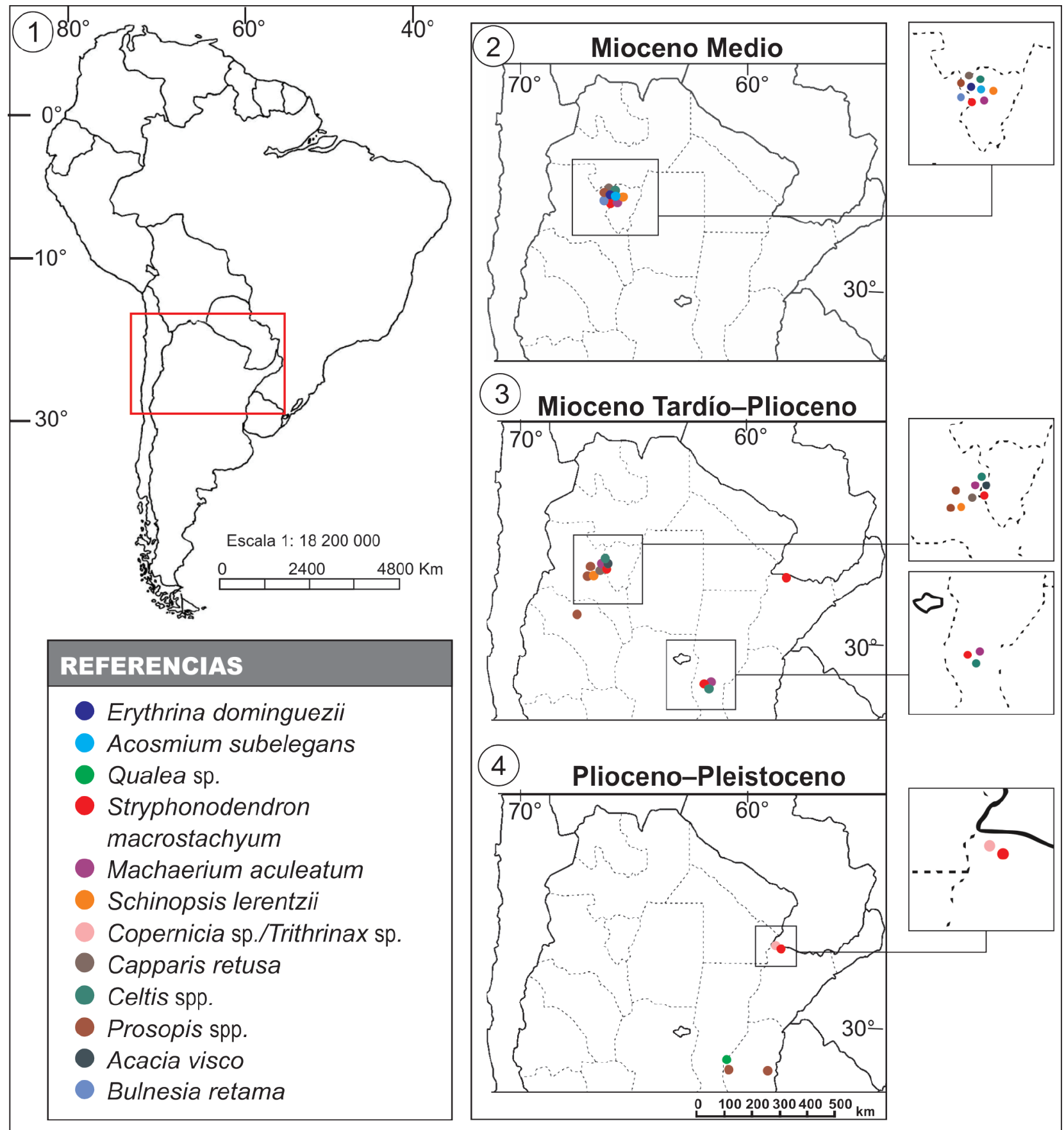

Figura 2. 1, Área generalizada de los yacimientos plantíferos neógenos de las unidades vegetales del Chaco y del Cerrado. 2, Distribución de las especies fósiles en el Mioceno Medio. 3, Distribución de las especies fósiles en el Mioceno Tardío-Plioceno. 4, Distribución de las especies fósiles en el Pleistoceno.

gistro fuera de los actuales límites establecidos para ellos. Por otra parte, dado que la vegetación del Cerrado se localiza en el planalto brasileño y zonas aledañas (centro de Brasil) (Fig. 3), puede interpretarse como una retracción de esta paleovegetación desde el Mioceno hasta la actualidad.

A fin de comprender la evolución y los cambios de las asociaciones florísticas mencionadas en esta contribución, los trabajos de Hinojosa y Villagrán (1997) y Barreda et al. 


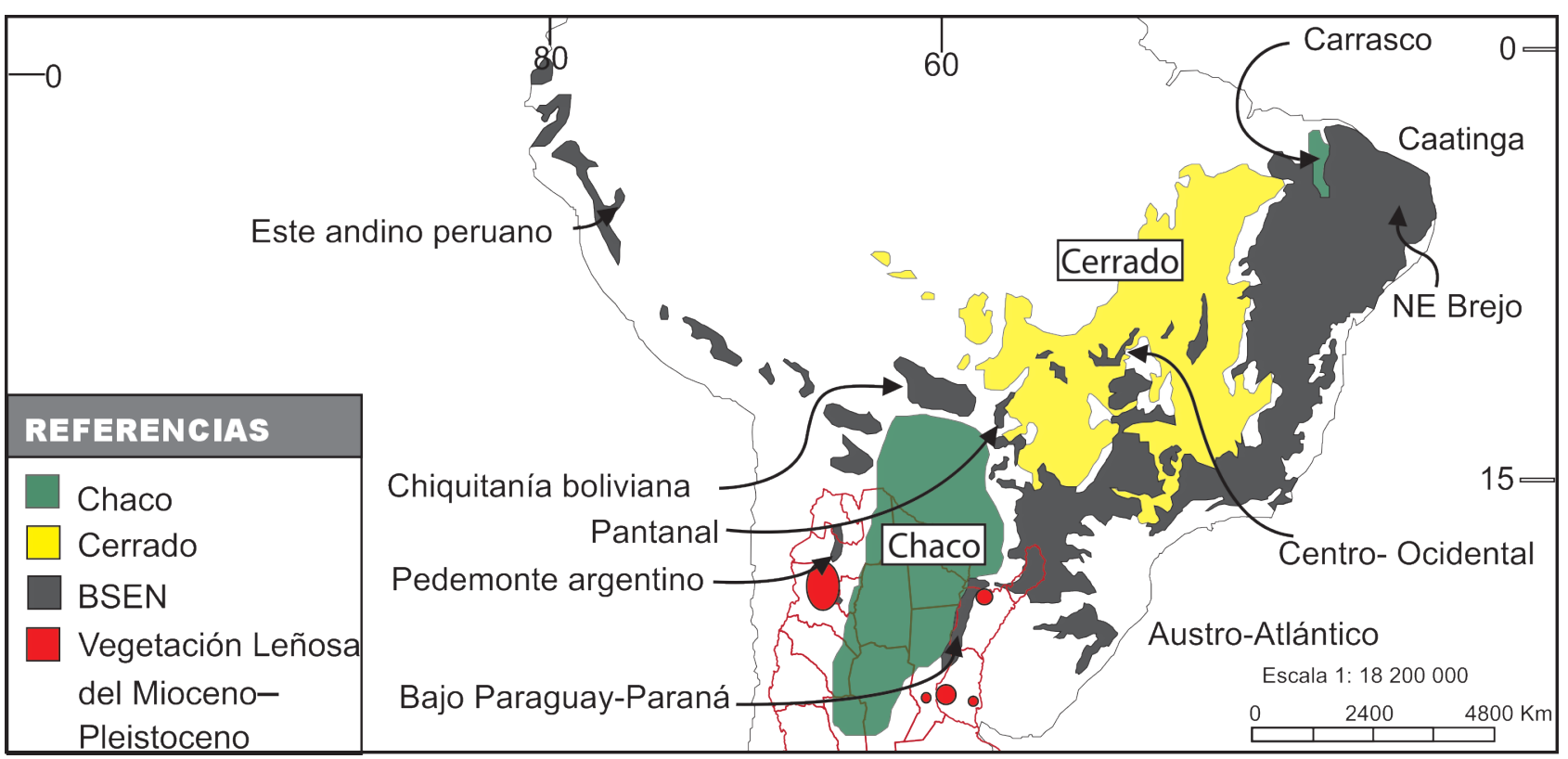

Figura 3. Distribución geográfica de los BSEN (Bosques estacionales secos neotropicales), de las unidades vegetales del Chaco y del Cerrado y de la vegetación fósil (adaptado de Mogni et al., 2015).

(2007) tratan la evolución de la paleoflora neógena del cono sur y las causas de sus modificaciones. Estos cambios paleovegetacionales habrían obedecidos a diversos factores, entre ellos: los cambios orbitales, las variaciones en las concentraciones atmosféricas de CO2, la separación de la Antártida de Sudamérica y la apertura del estrecho de Drake con la consiguiente instalación de la corriente circumpolar, la expansión de las masas de hielo antárticas, los inicios de las glaciaciones y la elevación de la cordillera de los Andes, los que habrían provocado una abrupta caída del nivel del mar. Este conjunto de factores llevaron al desmejoramiento climático (disminución de la humedad ambiental con periodos fríos y aridez) y a la instalación progresiva de la vegetación xerófila. Por otra parte, se debe destacar que la Ingresión Marina Entrerriense-Paranaense (Mioceno Medio-Tardío) habría influido en la distribución de las asociaciones florísticas aquí tratadas.

\section{CONCLUSIÓN}

En esta contribución se confirma la existencia de asociaciones leñosas neógenas en el centro norte de Argentina antecesoras a los BSEN, a la vegetación del Chaco y del Cerrado. Si bien algunos representantes de los BSEN estaban ya presentes en el Mioceno Medio, esta asociación florística leñosa tuvo una diversificación notoria entre el Mioceno Tardío y Plioceno, siendo su distribución geográfica fragmentaria y disyunta, entre el noroeste y noreste, semejante a la actual. También se confirma la hipótesis planteada por Pennington et al. (2004) de que la radiación de las especies con alto grado de endemismo en los BSEN, ocurrió durante el Mioceno Tardío y Plioceno. Por otra parte las especies fósiles afines a la actual vegetación leñosa del Chaco y Cerrado muestran una diversificación más temprana, especialmente desde el Mioceno Medio. Se observa además, que particularmente los antecesores fósiles del Cerrado tuvieron una distribución geográfica más amplia y austral de la que se manifiesta en el presente y que los BSEN habrían tenido una retracción parcial tanto en el noroeste como en el noreste. Esta distribución habría estado influenciada por la Ingresión Marina Entrerriense-Paranaense producida entre Mioceno Medio a Tardío. Por último, las asociaciones paleoflorísticas aquí tratadas habrían sido el producto del movimiento de placas tectónicas y procesos atmosféricos, entre otros, que llevaron al desmejoramiento climático (disminución de la humedad ambiental, periodos fríos y aridez) y a la instalación progresiva de la vegetación xerófila. 


\section{AGRADECIMIENTOS}

El presente trabajo se realizó en el marco de los siguientes proyectos: PIP N 11220110100417, CONICET y PI 16F008, Secretaría de Ciencia y Técnica de la UNNE. D. Prado agradece a la Universidad Nacional de Rosario, al CONICET y al IICAR (CONICET-UNR). Las autoras agradecen a M. Bechara por el asesoramiento y confección de las figuras.

\section{REFERENCIAS}

Anzótegui, L.M. 2002. Hojas de Myrtaceae en la Formación San José (Mioceno medio) Provincia de Tucumán, Argentina. Ameghiniana 39: 321-330.

Anzótegui, L.M., Garralla, S.S. y Herbst, R. 2007a. Fabaceae de la Formación El Morterito, (Mioceno superior) del valle del Cajón, provincia de Catamarca, Argentina. Ameghiniana 44: 183-196.

Anzótegui, L.M. y Herbst, R. 2004. Megaflora (hojas y frutos) Santa María, provincia de Catamarca, Argentina. Ameghiniana 41: 423436.

Anzótegui, L.M. y Horn, Y. 2011. Megaflora de la Formación Palo Pintado (Mioceno superior) Salta, Argentina. Parte II. Revista brasileira de paleontologia 14: 239-254.

Anzótegui, L.M., Horn, Y. y Herbst, R. 2007b. Paleoflora (Fabaceae y Anacardiaceae) de la Formación Andalhuala (Plioceno Inferior), provincia de Catamarca, Argentina. Ameghiniana 44: 525-535.

Anzótegui, L.M. y Lutz, A.L. 1988. Paleocomunidades vegetales del Terciario superior (Formación Ituzaingó) de la Mesopotamia Argentina. Revista de la Asociación de Ciencias Naturales del Litoral 18: 105-228.

Anzótegui, L.M., Mautino, L.R., Garralla, S.S., Herbst, R., Robledo, J.M. y Horn, M.Y. 2017. Paleovegetación cenozoica del noroeste argentino. En: M. Muruaga y P. Grosse (Eds.), Ciencias de la tierra y recursos naturales del NOA: relatorio del $20^{\circ}$ Congreso Geológico Argentino. Asociación Geológica Argentina, Tucumán, p. 763777.

Barreda, V.D., Anzótegui, L.M., Prieto, A.R., Aceñolaza, P., Bianchi, M.M., Borromei, A.M., Brea, M., Caccavari, M., Cuadrado, G.A., Garralla, S., Grill, S.G., Guerstein, R., Lutz, A.I., Mancini, M.V., Mautino, L.R., Ottone, E.G., Quattrocchio, M., Romero, E.J., Zamaloa, M.C. y Zucol, A. 2007. Diversificación y cambios de las Angiospermas durante el Neógeno en Argentina. Publicación Especial de la Asociación Paleontológica Argentina 11: 173-191.

Barreda, V.D. y Caccavari, M. 1992. Mimosoideae (Leguminosae) occurrence in the Early Miocene of Patagonia (Argentina). Palaeogeography, Palaeoclimatology, Palaeoclimatology 94: 243252.

Brea, M., Aceñolaza, P.G. y Zucol, A.F. 2001. Estudio paleoxilológico en la Formación Paraná, Entre Ríos, Argentina. Publicación Especial de la Asociación Paleontológica Argentina 8: 7-17.

Brea, M. y Franco, M.J. 2013. El registro fósil de Anacardiaceae en el Cenozoico tardío del Noreste Argentino. Acta Geológica Lilloana 25: 21-36

Brea, M., Zucol, A.F. y Patterer, N. 2010. Fossil woods from late Pleistocene sediments from El Palmar Formation, Uruguay Basin, Eastern Argentina. Review of Palaeobotany and Palynology 163: 35-51.

Caetano, S., Prado, D., Pennington, R.T., Beck, S., Oliveira-filho, A., Spichiger, R. y Naciri, Y. 2008. The history of Seasonally Dry Tropical Forests in eastern South America: inferences from the genetic structure of the tree Astronium urundeuva (Anacardiaceae). Molecular Ecology 17: 3147-3159.

DRYFLOR, 2016. Plant diversity patterns and their conservation implications in neotropical dry forests. Science 353: 1383-1387.
DRYFLOR, 2017. Forest conservation-Remember Gran ChacoResponse. Science 355: 465-466.

Fernández Pacella, L. 2015. Registro fósil y distribución de Anadenanthera en Argentina desde el Mioceno hasta la actualidad. Estudios Geológicos 71: 1-9.

Franco, M.J. 2009. Leños fósiles de Anacardiaceae en la Formación Ituzaingó (Plioceno-Pleistoceno), Toma Vieja, Paraná, Entre Ríos, Argentina. Ameghiniana 46: 587-604.

Franco, M.J. 2010. Soroceaxylon entrerriensis gen. et sp. nov. (Moraceae) de la Formación Ituzaingó (Plioceno-Pleistoceno), Cuenca del río Paraná, Argentina. Revista Mexicana de Ciencias Geológicas 27: 508-519.

Franco, M.J. 2017. Small Celastraceae and Polygonaceae twigs from the Upper Cenozoic (Ituzaingo Formation) of the La Plata Basin, Argentina. Historical Biology 30: 1-15.

Franco, M.J y Brea, M. 2008. Leños fósiles de la Formación Paraná (Mioceno medio), Toma Vieja, Paraná, Entre Ríos, Argentina: registro de bosques estacionales mixtos. Ameghiniana 45: 699-717.

Franco, M.J. y Brea, M. 2009. Primer registro de leños fósiles de Moraceae y Rutaceae de la Formación Ituzaingó (Plioceno-Pleistoceno), Toma Vieja, Paraná, Argentina. $4^{\circ}$ Congreso Argentino de Cuaternario y Geomorfología, $12^{\circ}$ Congresso da Associação Brasileira de Estudos do Quaternário y II Reunión sobre el Cuaternario de América del Sur (La Plata), Actas p. 639-647.

Franco, M.J. y Brea, M. 2013. Leños fósiles de Leguminosas de la Formación Ituzaingó, Entre Ríos, Argentina: Implicancias paleoecológicas y paleobiogeográficas. Ameghiniana 50: 167-191.

Frederiksen, N.O. 1988. Sporomorphs, biostratigraphy, floral changes and paleoclimatology Eocene and earliest Oligocene of the eastern Gulf Coast. U. S. Geological Survey Professional Paper $1448,65 \mathrm{p}$.

Furley, P.A. 2007. Tropical savannas and associated forests: vegetation and plant ecology. Progress in Physical Geography 31: 203211.

Garralla, S.S., Anzótegui, L.M. y Mautino, L.R. 2016. Relaciones paleoflorísticas del Mioceno-Plioceno del norte argentino. Publicación Electrónica de la Asociación Paleontológica Argentina 16: $1-13$.

Gosling, W.D., Mayle, F.E., Tate, N.J. y Killeen, T.J. 2005. Modern pollen-rain characteristics of tall terra firme moist evergreen forest, southern Amazonia. Quaternary Research 64: 284-297.

Hinojosa, F.L. y Villagrán, C. 1977. Historia de los bosques del sur de Sudamérica, I: antecedentes paleobotánicos, geológicos y climáticos del Terciario del cono sur de América. Revista Chilena de Historia Natural 70: 225-239.

Horn, Y.M. 2014. [Palinofloras de las Formaciones El Morterito y Palo Pintado (Mioceno superior-Plioceno inferior), noroeste de Argentina. Tesis Doctoral, Facultad de Ciencias Naturales y Museo de La Plata, Universidad Nacional de La Plata, Buenos Aires, 142 p. Inédita.].

Martínez, L.C.A y Rodríguez-Brizuela, R. 2011. Gleditisioxylon riojana nov. sp. en el Neógeno de Argentina: sus relaciones paleoambientales y biogeográficas. Geobios 44: 461-472.

Mautino, L.R. 2010. [Palinoflora de las Formaciones San José y Chiquimil (Mioceno medio-superior) Noroeste de Argentina. Tesis Doctoral, Facultad de Ciencias Exactas y Naturales y Agrimensura, Universidad Nacional del Nordeste, Corrientes, 444 p. Inédita.].

Mautino, L.R. y Anzótegui, L.M. 2014. Novedades palinológicas en las formaciones San José y Chiquimil (Mioceno medio y superior) noroeste de Argentina. Revista del Museo de Ciencias Naturales 16: 143-164. 
Mautino, L.R., Garralla, S., Anzótegui, L.M., Galli, C. y Ramírez, F. 2017. Palinoflora de la secuencia basal de la Formación Palo Pintado (Mioceno Tardío-Plioceno) en la localidad Quebrada del Estanque, Salta, Argentina. $20^{\circ}$ Congreso Geológico Argentino y $4^{\circ}$ Simposio del Mioceno-Pleistoceno del Centro y Norte de Argentina (San Miguel de Tucumán), 62-64.

Mayle, F.E. 2004. Assessment of the Neotropical dry forest refugia hypothesis in the light of palaeoecological data and vegetation model simulations. Journal of Quaternary Science 19: 713-720.

Mayle, F.E., Beerling, D.J., Gosling, W.D. y Bush, M.B. 2004. Responses of Amazonian ecosystems to climatic and atmospheric carbon dioxide changes since the last glacial maximum. Philosophical Transactions of the Royal Society of London 359: 499-514.

McIntyre, D.J. 1965. Some new pollen species from New Zealand Tertiary deposits. New Zealand Journal of Botany 3: 204-214.

Mogni, V.Y., Oakley, L.J., Maturo, H.M., Galetti, L.A. y Prado, D.E. 2015. Biogeografía y Florística de los Bosques Secos Estacionales Neotropicales (BSEN). OKARA: Geografia em debate 9: 275-296.

Moya, E. y Brea, M. 2015a. A New record of fossil wood of Vochysiaceae from the Late Pleistocene (Arroyo Feliciano Formation) Argentina, South America. Revista brasilera de paleontología 18:83-90.

Moya, E. y Brea, M. 2015b. Legume fossil woods from the Arroyo Feliciano Formation (Late Pleistocene, northeastern Argentina): Paleobiogeographic implications. Ameghiniana 52: 558-573.

Moya, E., Brea, M. y Franco, M.J. 2015. First record of Styracoxylon (Styracaceae) from Southern Hemisphere: Arroyo Feliciano Formation (Upper Pleistocene), Entre Ríos, Argentina. Review of Palaeobotany and Palynology 221: 211-219.

Moya, E., Brea, M. y Lutz, A.I. 2017. Redescription and reassignment of the fossil wood Menendoxylon piptadiensis from the Pliocene Andalhuala Formation, South America. Journal of Systematic Palaeontology 16: 1-13.

Pennington, R.T., Lavin, M., Prado, D.E., Pendry, C.A., Pell, S. y Butterworth, C. 2004. Historical climate change and speciation: Neotropical seasonally dry forest plants show patterns of both Tertiary. Philosophical Transactions of the Royal Society, Series B, London 359: 515-538.

Pennington, R.T., Prado, D.E. y Pendry, C.A. 2000. Neotropical seasonally dry forests and quaternary vegetation changes. Journal of Biogeography 27: 261-273.

Pocknall, D.T. y Mildenhall, D.C. 1984. Late Oligocene-Early Miocene spores and pollen from Southland, New Zealand. New Zealand Geological Survey, Paleontological Bulletin 51: 1-66.
Prado, D.E. 1993a. What is the Gran Chaco vegetation in South America? I. A review. Contribution to the study of flora and vegetation of the Chaco. V. Candollea 48: 145-172.

Prado, D.E. 1993b. What is the Gran Chaco vegetation in South America? II. A redefinition. Contribution to the study of flora and vegetation of the Chaco. VII. Candollea 48: 615-629.

Prado, D.E. 2000. Seasonally dry forest of tropical South America: from forgotten ecosystems to a new phytogeographic unit. Journal of Botany 57: 437-461.

Prado, D.E. y Gibbs, P.E. 1993. Patterns of species distributions in the dry seasonal forests of South America. Annals of Misouri Botanical Garden 80: 902-927.

Pujana, R.R., Martínez, L.C.A., García Massini, J.L., Di lorio, O., Penas Steinhardt, A. 2014. Legume (Mimosoideae) fossil woods from the Late Miocene (Salicas Formation) of northwestern Argentina. Revista brasileira de paleontología 17: 317-326.

Ramos, R.S., Brea, M. y Kröhling, D.M. 2015. Primer registro de Cryptocaryoxylon Leisman, una Lauraceae de la Formación El Palmar (Pleistoceno tardío), Entre Ríos, Argentina. Revista del Museo Argentino de Ciencias Natutales 17: 59-70.

Ratter, J.A., Bridgewater, S. y Ribeiro, J.F. 2003. Analysis of the floristic composition of the Brazilian Cerrado vegetation III: Comparison of the woody vegetation of 376 areas. Edinburgh Journal of Botany 60: 57-109.

Ratter, J.A., Ribeiro, J.F. y Bridgewater S. 1997. The Brazilian Cerrado Vegetation and Threats to its Biodiversity. Annals of Botany 80 : 223-230.

Tropicos.org. Missouri Botanical Garden. World Wide Web: http://www.tropicos.org

Zuloaga, F.O., Morrone, O. y Belgrano, M.J. 2008. Catálogo de las plantas vasculares del Cono Sur (Argentina, sur de Brasil, Chile, Paraguay y Uruguay). Monograph Systematic Botany, Missouri Botanical Garden 107(2). World Wide Web: http://www2.darwin.edu.ar/ Proyectos/FloraArgentina/FA.asp

Doi: 10.5710/PEAPA.12.04.2019.279

Recibido: 13 de diciembre 2018

Aceptado: 12 de abril 2019 